OPEN ACCESS

Edited by:

Rami I. Aqeilan,

Hadassah Medical Center, Israel

Reviewed by:

Muhannad Mohammed Ali Abu-Remaileh,

Hebrew University of Jerusalem, Israel John P. Hagan,

University of Texas Health Science Center at Houston, United States

*Correspondence:

Séverine Lamon

severine.lamon@deakin.edu.au

Specialty section: This article was submitted to

RNA,

a section of the journal

Frontiers in Genetics

Received: 09 August 2018

Accepted: 26 October 2018

Published: 16 November 2018

Citation:

Mikovic J, Sadler K, Butchart L,

Voisin S, Gerlinger-Romero F,

Della Gatta P, Grounds MD and Lamon S (2018) MicroRNA and Long

Non-coding RNA Regulation

in Skeletal Muscle From Growth

to Old Age Shows Striking

Dysregulation of the Callipyge Locus.

Front. Genet. 9:548.

doi: 10.3389/fgene.2018.00548

\section{MicroRNA and Long Non-coding RNA Regulation in Skeletal Muscle From Growth to Old Age Shows Striking Dysregulation of the Callipyge Locus}

\author{
Jasmine Mikovic ${ }^{1}$, Kate Sadler ${ }^{1}$, Lauren Butchart', Sarah Voisin ${ }^{3}$, \\ Frederico Gerlinger-Romero ${ }^{1}$, Paul Della Gatta ${ }^{1}$, Miranda D. Grounds ${ }^{2}$ and \\ Séverine Lamon ${ }^{1 *}$
}

${ }^{1}$ School of Exercise and Nutrition Sciences, Institute for Physical Activity and Nutrition, Deakin University, Geelong, VIC, Australia, ${ }^{2}$ School of Human Sciences, The University of Western Australia, Perth, WA, Australia, ${ }^{3}$ Institute of Health and Sport, Victoria University, Footscray, VIC, Australia

MicroRNAs (miRNAs) undergo high levels of regulation in skeletal muscle development and control skeletal muscle mass, function and metabolism over the lifespan. More recently, the role of long non-coding RNAs (IncRNAs) in skeletal muscle regulation has started to emerge. Following up on our recent study describing the expression pattern and putative roles of 768 miRNAs in the quadriceps muscle of mice at early life stages, we used a high-throughput miRNA qPCR-based array to assess the expression of the same miRNAs in 28-month old male mouse quadriceps muscle. In addition, we report the expression patterns of IncRNAs playing a putative role in muscle development and adaptation from growth to old age. Twelve miRNAs were significantly downregulated in 28-month old muscle when compared with 12-week old muscle. Ten of them clustered at the DIk1-Dio3 locus, known as 'Callipyge,' which is associated with muscle development and hypertrophy. This collective downregulation was paralleled by decreases in the expression levels of the maternally expressed imprinted LncRNA coding genes Meg3 and Rian stemming from the same chromosomal region. In contrast, the paternally expressed imprinted Dlk1-Dio3 locus members Rt/1, Dio3, and DIk1 and the muscle related IncRNAs IncMyoD1, Neat_v1, Neat_v2, and Malat1 underwent significant changes during growth, but their expression levels were not altered past the age of 12 weeks, suggesting roles limited to hyperplasia and early hypertrophy. In conclusion, collective muscle miRNA expression gradually decreases over the lifespan and a cluster of miRNAs and maternally expressed IncRNAs stemming from the Callipyge locus is significantly dysregulated in aging muscle. The Dlk1-Dio3 locus therefore represents a potential new mechanism for age-related muscle decline.

Keywords: aging, miRNAs, IncRNAs, sarcopenia, skeletal muscle 


\section{INTRODUCTION}

Maintaining skeletal muscle mass and function is paramount to preserve mobility and whole-body metabolism over the lifespan. Skeletal muscle mass and function naturally declines from maturity to old age (Lexell et al., 1988; Sayer et al., 2013). This occurs as a consequence of the gradual dysregulation of a number of processes that are essential to maintain skeletal muscle structure, metabolism and mitochondrial function (Rooyackers et al., 1996; Short et al., 2005; Birben et al., 2012). Decreased protein synthesis and increased protein degradation are observed in aging humans and rodents (Combaret et al., 2005; Rieu et al., 2006) and lead to a net loss of protein that, in conjunction with a progressive loss of innervation of aging myofibers (Chai et al., 2011), result in age-related muscle atrophy and loss of function (Barns et al., 2014; White et al., 2016b). Muscle regeneration relies on the efficiency of a population of quiescent muscle stem cells, called satellite cells, to activate, proliferate, differentiate and fuse (Yin et al., 2013). Muscle regeneration capacity generally decreases with aging (Yun, 2015), although a central role for intrinsic decline of old satellite cell response is disputed (Grounds, 2014). Elevated Wnt (Fujimaki et al., 2015) and decreased Notch1 (Carey et al., 2007) may contribute to reduced regenerative capacity. Canonical Wnt signaling, essential for satellite cell differentiation and self-renewal (von Maltzahn et al., 2012), inhibits myogenic regeneration and promote muscle progenitor cells to a fibrogenic fate over a myogenic fate (Fujimaki et al., 2015). While the extrinsic age-related mechanisms involved in regeneration are not fully understood, clear alterations are seen in the aging immune system (Jackaman et al., 2013) with a delayed inflammatory response to muscle injury (Grounds, 2014). The aged systemic environment also modifies the extracellular matrix, with a fibrotic environment altering satellite cell behavior and myogenic capacity (StearnsReider et al., 2017). Finally, reduced mitochondrial enzyme activity and increased reactive oxygen species levels induce irreversible damage to DNA and other macromolecules, altering cell function (Rooyackers et al., 1996; Short et al., 2005; Birben et al., 2012; Tohma et al., 2014). Hence a wide range of factors collectively contribute to a decline in muscle mass and function with advancing age, termed 'sarcopenia.' In the mouse, the onset of sarcopenia occurs between 15 and 18 months of age (Barns et al., 2014; White et al., 2016b) and peaks at 24 months of age (Barns et al., 2014; Soffe et al., 2016).

Muscle homeostasis is fine-tuned by the coordinate action of muscle-specific transcription factors and epigenetic regulators. These include DNA methylation, histone modification (Asp et al., 2011; Howlett and McGee, 2016), as well as the non-coding RNA molecules microRNAs (miRNAs) (Zhao and Srivastava, 2007) and long non-coding RNAs (lncRNAs) (Wang and Chang, 2011), which are the focus of this study. MiRNAs are 2024 nucleotides single-stranded RNA molecules that make up $1-3 \%$ of the eukaryote genome (Zhao and Srivastava, 2007). MiRNAs can be tissue specific and are mostly conserved between species (Kovanda et al., 2014). They regulate gene and protein expression, mostly through degrading messenger RNA (mRNA) or inhibiting protein translation (Bartel, 2004). Skeletal muscle enriched miRNAs, termed myomiRs, include miR-1, miR-133a/b and miR-206 (McCarthy, 2008) and have important and partly redundant functions in regulating satellite cell proliferation, differentiation and apoptosis through fine-tuning Paired Box (PAX) and myogenic regulatory factor (MRF) genes (Huang et al., 2012; Kirby and McCarthy, 2013). However, hundreds of other miRNAs are expressed in skeletal muscle tissue and are subjected to substantial levels of regulation during muscle development (Lamon et al., 2017).

Long non-coding RNAs are classified as non-coding RNA molecules exceeding 200 nucleotides (Engreitz et al., 2016). Like miRNAs, lncRNAs are tissue specific, however, they are poorly conserved between species (Johnsson et al., 2014). It was long thought that lncRNAs lacked any functional roles. Recent findings, however, suggest that lncRNAs have multiple modes of gene regulation and can act as signal, guide, scaffold and decoy molecules for other RNA species, including mRNAs and miRNAs (Wang and Chang, 2011). The role of lncRNAs in skeletal muscle growth (Neguembor et al., 2014) and aging has begun to emerge (Neppl et al., 2017; Zong-Kang et al., 2018). For example, Chronos is a lncRNA that increases with age in mouse muscle and impairs skeletal muscle hypertrophy through repressing Bmp7 (Neppl et al., 2017). The expression of another muscle enriched lncRNA, muscle anabolic regulator 1 (MAR1), also decreases with advancing age in mice and might promote muscle differentiation and regeneration through regulating myogenic mediator Wnt5a (Zong-Kang et al., 2018).

We recently observed that a series of lncRNAs, including lncRNA Metastasis associated lunch adenocarcinoma transcript 1 (Malat1), the two variants of Nuclear Enriched Abundant Transcript 1 (Neat1_v1 and Neat1_v2), long non-coding MyoD (LncMyoD) and Lincmd1 underwent significant dysregulation in early post-natal murine muscle growth (Butchart et al., 2016). Malat1 and Neat1 have putative roles in muscle cell differentiation (Sunwoo et al., 2009; Watts et al., 2013; Butchart et al., 2016). Canonical MRF MyoD activates LncMyoD during myoblast differentiation (Gong et al., 2015), suggesting that LncMyoD plays a role in promoting cell cycle exit and commitment to differentiation. This process is fine-tuned by long intergenic non-coding Lincmd1, which regulates the timing of myoblast differentiation (Cesana et al., 2011), while also acting as an endogenous sponge for the canonical myomiR miR-133 (Cesana et al., 2011). Research as to whether lncRNAs plays a direct role in facilitating age-related muscle decline is, however, rudimentary.

This study follows on from our recently published work that identified collective miRNA (Lamon et al., 2017) and LncRNA (Butchart et al., 2016) expression in mouse quadriceps muscle over the first 12 weeks of age. This study in young growing mice (up to 3 months) has expanded to analyze muscle from very old mice (aged 28 months old), and examines the expression of 768 miRNAs and selected lncRNAs in mouse quadriceps muscle. Comparing miRNA and LncRNA expression patterns in muscle tissue between such young and old stages of the lifespan is important to increase our understanding of the biological roles of these complex molecules in age-related loss of muscle mass and function, with possible identification of novel therapeutic targets. 


\section{MATERIALS AND METHODS}

\section{Mouse Muscle Samples}

All animals (male C57BL/6J) were purchased from the Animal Resource Centre, Murdoch, Western Australia. Animal experiments were approved by the Animal Ethics committees at the University of Western Australia (RA/3/100/1436); La Trobe University (AEC15-32); and Deakin University (G032015). Animal experiments were conducted in strict accordance with guidelines outlined in the National Health and Medical Research Council Code of practice for the care and use of animals for scientific purposes (2004), and the Animal Welfare act of Western Australia (2002). All mice were housed under standard humidity, temperature and lighting conditions, and had ad libitum access to food and drinking water. Mice sacrificed at 2 days $(n=4), 2,4$, and 12 weeks of age $(n=6$ per age group) were housed at the University of Western Australia pre-clinical facility as described previously (Lamon et al., 2017). Mice sacrificed at 28 months of age $(n=11)$ were housed at La Trobe University animal facility and sacrificed by decapitation. Quadriceps muscles were dissected out and snap frozen in liquid nitrogen before being stored at $-80^{\circ} \mathrm{C}$.

\section{RNA Extraction and Reverse Transcription}

Total RNA was extracted from the quadriceps muscle as previously described (Lamon et al., 2017). Total RNA was treated with DNase I Amplification Grade (Thermo Fisher Scientific, Waltham, MA, United States) and total RNA concentration was assessed using the NanoDrop 1000 Spectrophotometer (Thermo Fisher Scientific). For miRNA analysis, RNA (500 ng) was reversed transcribed using the TaqMan MicroRNA Reverse Transcription (RT) kit and Megaplex RT Primers, Rodent Pool A and Pool B v3.0 (Thermo Fisher Scientific) as previously described (Lamon et al., 2017). For mRNA and lncRNA expression analysis, first-strand cDNA was generated was generated from RNA (500 ng) using the High Capacity cDNA Reverse Transcription Kit (Thermo Fisher Scientific) as previously described (Lamon et al., 2017).

\section{Single-Strand DNA Quantification}

First-strand cDNA was generated as described above. cDNA was then treated with RNase H (Thermo Fisher Scientific) according to the manufacturer's protocol. Single-strand DNA was quantified using the Quant it OliGreen ssDNA Assay Kit (Thermo Fisher Scientific) according to the manufacturer's instructions and used for mRNA and lncRNA PCR normalization.

\section{MiRNA Screening and Target Analysis}

MicroRNA expression was assessed using the TaqMan Array Rodent MicroRNA A + B Cards v3.0 (Thermo Fisher Scientific) as described previously (Zacharewicz et al., 2014; Lamon et al., 2017). The results from the Megaplex were analyzed using the ExpressionSuite Software v1.0 (Thermo Fisher Scientific) and the data were normalized using the global normalization function, which we previously identified as the technique that most accurately represents biological variations (Zacharewicz et al., 2014; Lamon et al., 2017; Russell et al., 2017). Ct values were transformed into arbitrary units (AU) using the following equation: $\mathrm{AU}=(1 / 2)^{\mathrm{Ct}} 10^{10}$ and expressed relative to the mean value of the latest time point (28 months). Target analysis was performed as described previously (Zacharewicz et al., 2014; Lamon et al., 2017).

\section{Real-Time PCR}

Real-time PCR was carried out using a Stratagene MX3000 thermal cycler (Thermo Fisher Scientific). mRNA and lncRNA levels were measured using $1 \times \mathrm{SYBR}^{\otimes}$ Green PCR Master Mix (Thermo Fisher Scientific) and $6.25 \mathrm{ng}$ of cDNA. All primers were used at a final concentration of $300 \mathrm{nM}$, with the exception of Qiagen QuantiTect (QT) primers that were used according to the manufacturer instructions. The PCR conditions have been previously described for mRNAs (Lamon et al., 2017) and lncRNAs (Butchart et al., 2016), with specific melting temperatures shown in Table 1. mRNA and lncRNA levels were normalized to total cDNA input. Primer sequences and characteristics are presented in Table $\mathbf{1}$ and in Lamon et al. (2017).

\section{Protein Extraction and Western Blotting}

Total protein was extracted using RIPA buffer (Millipore, North Ryde, NSW, Australia) with $10 \mu \mathrm{L} / \mathrm{mL}$ Phosphatase and Protease Inhibitor Cocktail (Thermo Fisher Scientific). Total protein content was determined using the BCA Protein Assay Kit (Pierce Biotechnology, Rockford, IL, United States) according to the manufacturer's instructions. The proteins were separated out using a $4-15 \%$ gradient Criterion TGX (tris-glycine) Stain Free gel (Bio-Rad, Hercules, CA, United States) and transferred to a PVDF membrane. The membranes were blocked in 5\% skim milk in TBST for $1 \mathrm{~h}$ at room temperature, after which they were incubated overnight at $4^{\circ} \mathrm{C}$ with a $\mathrm{DIO} 3$ antibody (Biorbyt, 213851) diluted in 5\% BSA in TBST at 1:500. Following overnight incubation, the membranes were washed in TBST and incubated for $1 \mathrm{~h}$ at room temperature with an anti-rabbit IgG antibody labeled with an infrared fluorescent $800 \mathrm{~nm}$ dye (Alexa Fluor ${ }^{\circledR}$ 800; Thermo Fisher Scientific) diluted 1:5000 in $5 \%$ BSA in TBST. After washing, the proteins were exposed on an Odyssey ${ }^{\circledR}$ CLx Infrared Imaging System (LI-COR Biosciences) and individual protein band optical densities were determined using the Odyssey ${ }^{\circledR}$ Infrared Imaging System software. All blots were normalized against total protein load using the Bio-Rad Image Lab software (v6.0).

\section{Statistical Analysis}

All data are reported as mean \pm SEM. Diagnostic plots of residuals and fitted values were checked to ensure homogeneity of variance and unpaired Student's $t$-tests or one-way analysis of variance (ANOVA) were used to compare group means (GenStat v18). Adjustment for multiple testing was performed by using the Bonferroni correction that allows controlling the overall falsepositive rate for all inferences and is recognized as the most stringent when the various tests being performed are not highly correlated. The significance level was set at $p<0.05$. 
TABLE 1 | Mouse primers for real time PCR analysis.

\begin{tabular}{|c|c|c|c|c|}
\hline Gene & GenBank accession \# & Forward primer $\left(5^{\prime}-3^{\prime}\right)$ & Reverse primer $\left(5^{\prime}-3^{\prime}\right)$ & Melting temp \\
\hline$R t / 1$ & NM_184109.2 & TACTGCTCTTGGTGAGAGTGGACCC & GGAGCCACTTCATGCCTAAGACGA & $57^{\circ} \mathrm{C}$ \\
\hline$D \mid k 1$ & NM_010052.5 & TACCCCTAACCCATGCGAGA & GCACAGACACTCGAAGCTCA & $55^{\circ} \mathrm{C}$ \\
\hline Dio3 & NM_172119.2 & CCACGTGCAAATGCTCCAAA & TCAGTTCGAGCCACAGCAAT & $55^{\circ} \mathrm{C}$ \\
\hline Mef2a & NM_001033713.2 & GCGGAGACTCGGAATTGCAT & GGCTGCCGTTGAAATTGTCT & $55^{\circ} \mathrm{C}$ \\
\hline LncRNA & GenBank accession \# & Forward primer $\left(5^{\prime}-3^{\prime}\right)$ & Reverse primer $\left(5^{\prime}-3^{\prime}\right)$ & Melting temp \\
\hline LncMyoD & $\mathrm{N} / \mathrm{A}$ & GCAAGAAAACCACAGAGGAGG & GTGAAGTCCTTGGAGTाGAG & $60^{\circ} \mathrm{C}$ \\
\hline Neat1_v1 (short) & NR_003513.3 & ACCCIIITCATGGGGGTAG & GCTGGATGGAGGCTTGTTA & $55^{\circ} \mathrm{C}$ \\
\hline Neat1_v2 (long) & NR_131212.1 & GCTCTGGGACCTTCGTGACTCT & CTGCCTTGGCTTGGAAATGTAA & $55^{\circ} \mathrm{C}$ \\
\hline Malat1 & NR_002847.3 & ATGTCTCCATGGGGAATGAG & TATGCAGCTITCATCAGTAGGA & $60^{\circ} \mathrm{C}$ \\
\hline Lincmd1 & NR_131249.1 & CTGAAGGACACAAGGTGGCTT & AACTGAGGCTCCCAGTAAGA & $60^{\circ} \mathrm{C}$ \\
\hline AK050713 & $\mathrm{N} / \mathrm{A}$ & TGTTAGGTGCCTTCTCTGCGTGC & GCTGACGCTTCCCTGACAATCTTGTAG & $55^{\circ} \mathrm{C}$ \\
\hline LncRNA & GenBank accession \# & Company (catalog number) & Melting temp & \\
\hline Rian1 (Meg8) & $\begin{array}{l}\text { NR_028261, } \\
\text { XM_901568, } \\
\text { XM_922645 }\end{array}$ & Qiagen (QT01197784) & $55^{\circ} \mathrm{C}$ & \\
\hline Gt/2 (Meg3) & \multicolumn{2}{|c|}{$\begin{array}{l}\text { NM_144513, NR_003633, NR_027651, NR_027652, } \\
\text { NR_046475, XM_981786, XR_035483, XR_035484 }\end{array}$} & Qiagen (QT00161658) & $55^{\circ} \mathrm{C}$ \\
\hline Mirg (Meg9) & \multicolumn{2}{|l|}{ XM_488655 } & Qiagen (QT00285747) & $55^{\circ} \mathrm{C}$ \\
\hline
\end{tabular}

\section{RESULTS}

\section{Collective Expression Patterns of miRNAs in Aging Quadriceps Muscle}

Our first investigation of miRNA expression patterns with aging compared young adult aged 12 weeks with geriatric 28month male C57Bl/6J mice. The cut-off for the relevant level of expression of each miRNA was set at Mean (Ct) $<32$ for each time point, as recommended by the manufacturer. Out of the 768 miRNAs measured, 230 (30\%) were expressed at one time point at least (12 weeks or 28 months). A total of 101 miRNAs returned a $p$-value of $p<0.05$ before adjustment for multiple comparisons (Supplementary File S1). To assess whether a specific trend in expression change was associated with aging, preliminary analysis was conducted on this set of 101 miRNAs. Fold-change was calculated as the ratio of normalized miRNA expression level at the two time points. Overall, 42 miRNAs were downregulated and 59 miRNAs were upregulated at 28 months of age (Figure 1), confirming that individual miRNA expression can be positively or negatively affected by aging.

\section{Changes in Individual miRNAs in Aging Quadriceps Muscle}

To assess individual changes in miRNA expression in 12-week (young) and 28-month (old) quadriceps muscle, $p$-values were adjusted for multiple comparisons using the Bonferroni correction (Supplementary File S1). The expression levels of 12 miRNAs (mmu-miR-434-3p, mmu-miR-376c-3p, mmu-miR127-3p, hsa-miR-299-5p, mmu-miR-539-5p, mmu-miR-335-5p,

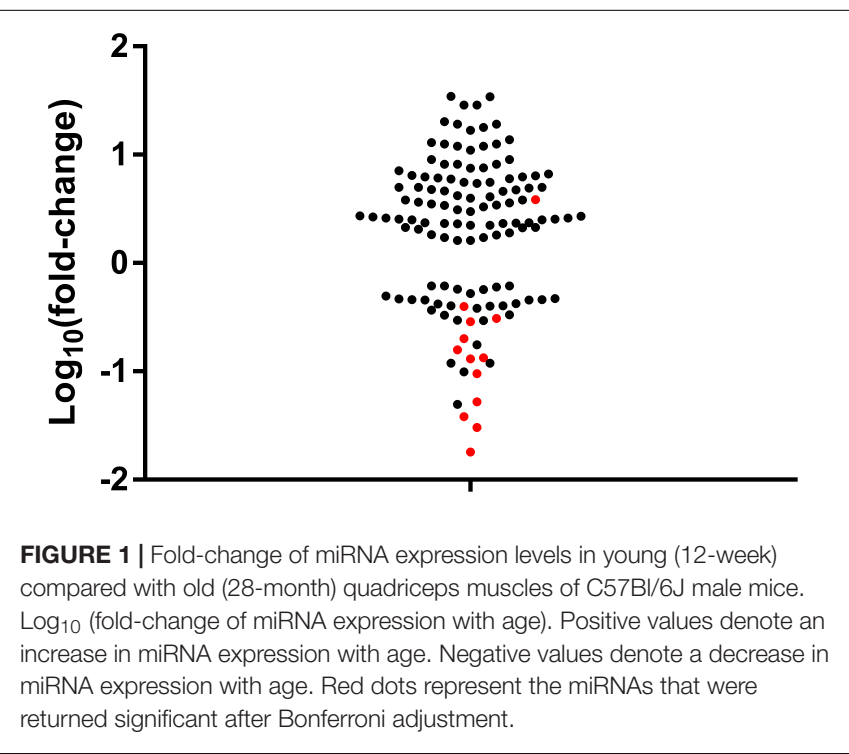

mmu-miR-1905, mmu-miR-136-5p, mmu-miR-532-5p, mmumiR-411-5p, mmu-miR-337-5p, and mmu-miR-382) were significantly different in old when compared with young muscle (Figure 2). All miRNAs, except for mmu-miR-1905, were downregulated with aging, with one additional miRNA, mmu-miR-673-3p, detected only in young (12-week) but not in old (28-month) mouse muscle (data not shown). Ten of these 12 downregulated miRNAs co-localized on two adjacent chromosomic clusters $(<30 \mathrm{~kb})$ located on chromosome 12: one cluster containing mmu-miR-127, mmu-miR-136, mmu-miR-337, mmu-miR-434 and mmu-miR-673; and 

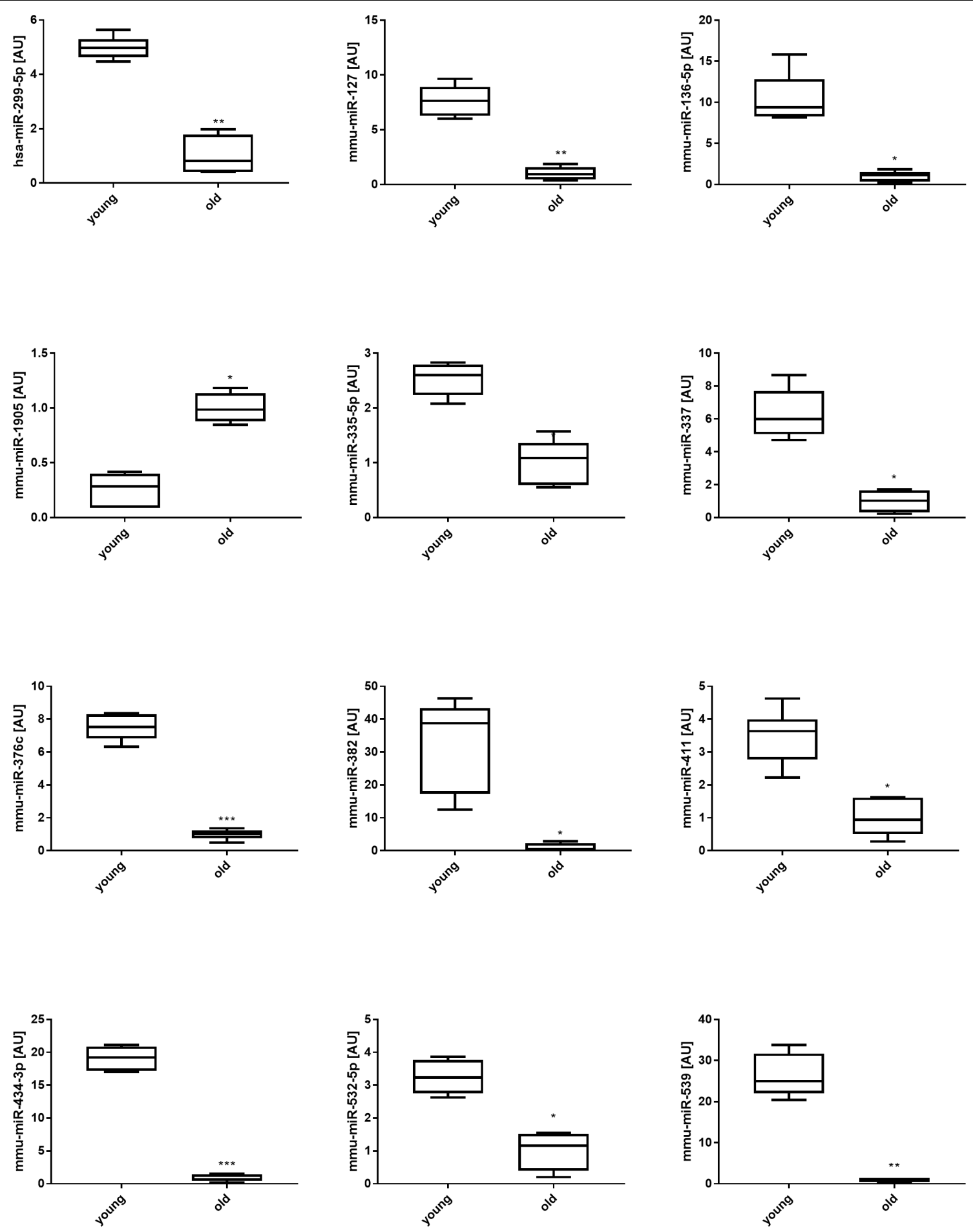

FIGURE 2 | Expression levels of individual miRNAs in young (12-week) compared with old (28-month) muscles. Individual miRNA expression levels are shown for 12 miRNAs that were significantly different in 28-month, when compared with 12-week, old mouse muscle. The data are reported as Mean \pm SEM. * $p<0.05$; ${ }^{* *} p<0.01$; and ${ }^{* * *} p<0.001$.

one cluster containing hsa-miR-299-5p, mmu-miR-376c, mmu-miR-382, mmu-miR-539 and mmu-miR-411 (Figure 3). LncRNA encoding genes Meg3 (Glt2), Mirg (Meg9), and Rian, and protein encoding genes $R t l 1, D i o 3$, and $D l k 1$ stem from the same chromosomal region, termed the Dlk1-Dio3 locus. These genes were therefore selected for further investigation. 


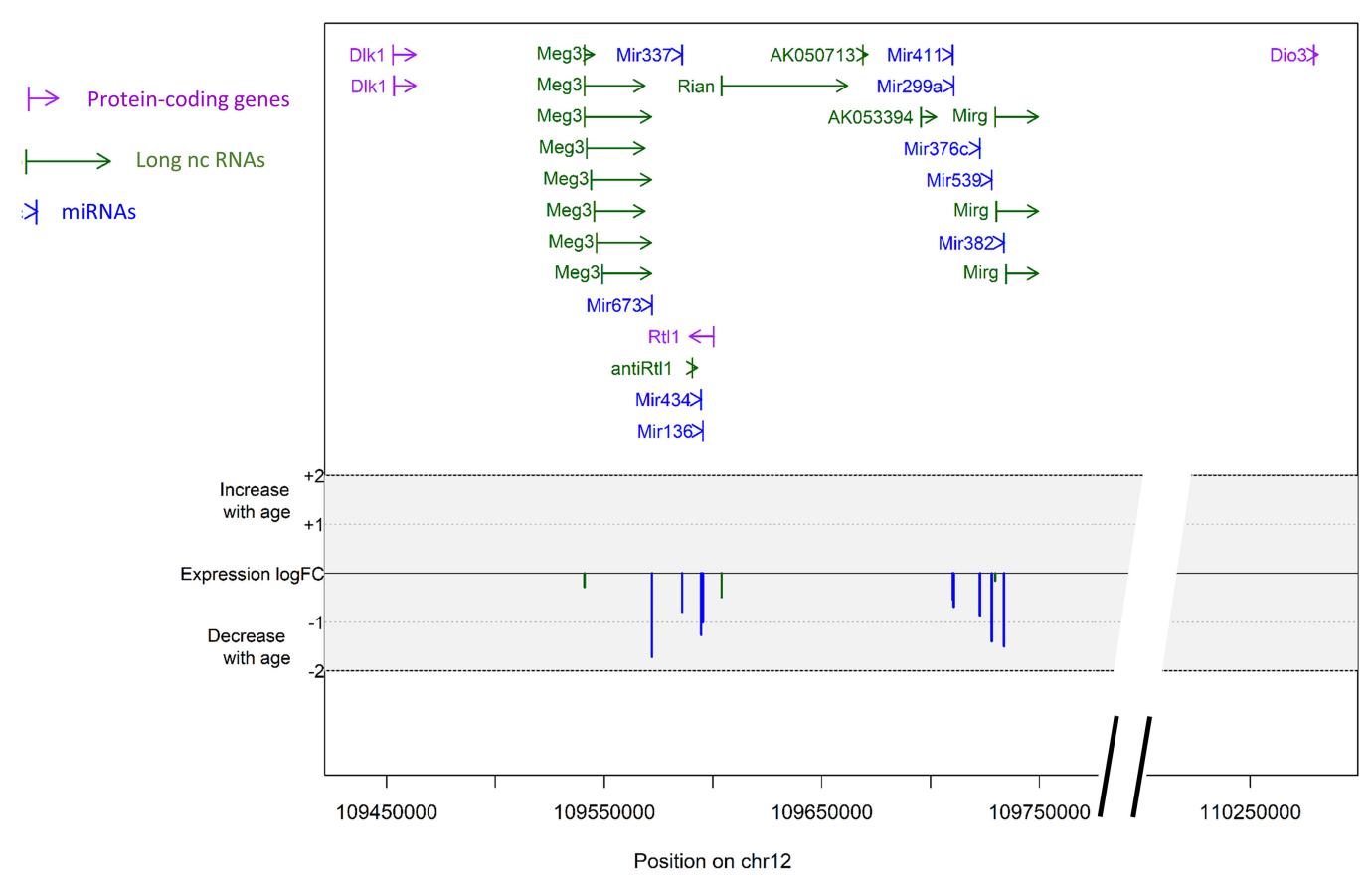

FIGURE 3 | Genomic context of the differentially expressed mRNAs, miRNAs, and IncRNAs in young (12-week) and old (28-month) muscle. We used the basic annotation of GENCODE mouse vM16 to annotate protein-coding genes (mRNAs) (purple), long non-coding genes (IncRNAs) (green), and miRNAs (blue), visible on the top panel. Differential expression between 12-week old and 28-month old mice for a given gene is visible as a vertical bar in the middle panel (log fold-change of expression). All transcripts had decreased expression with age. There was no difference in mRNA expression for the genes D/k1, Rt/1, and Dio3 between young (12-week) and old (28-month) muscles. This graph was created using an in-house R script. FC, fold change.
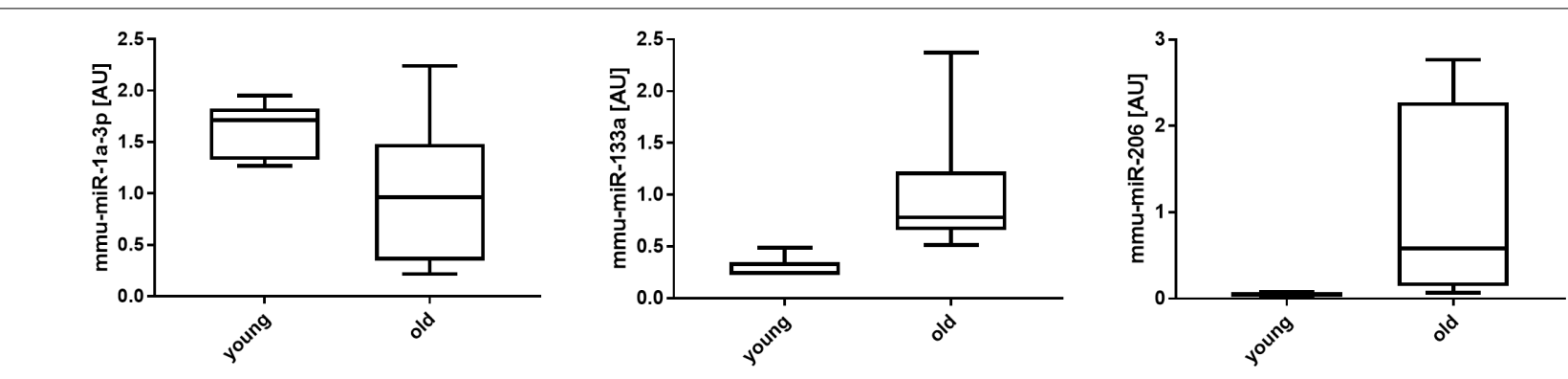

FIGURE 4 | MyomiRs expression levels in young (12-week) compared with old (28-month) muscles. Individual miRNA expression levels are shown for mmu-miR-1-3p, mmu-miR-133a-3p, and mmu-miR-206 in 28-month, when compared with 12-week, old mouse muscle. The data are reported as Mean \pm SEM.

\section{Changes in myomiR Expression in Aging Quadriceps Muscle}

The expression levels of the canonical myomiRs, -miR-1-3p, mmu-miR-133a-3p and mmu-miR-206, in 12-week (young) and 28-month (old) muscle are depicted in Figure 4. None of the myomiRs displayed a significant difference in old when compared with young muscle.

\section{Changes in miRNA and IncRNA Expression Over the Lifespan (Comparison of 5 Ages)}

In a prior study, we reported the individual and collective expression levels of miRNAs (Lamon et al., 2017) and lncRNAs
(Butchart et al., 2016) during the post-natal rapid growth phase of the quadriceps muscle, at 2 days, 2, 4, and 12 weeks after birth. MiRNAs were clustered using a robust quadratic model. These ages were then compared with the old muscle (aged 28 months). Out of the 13 miRNAs differentially regulated with aging, five miRNAs (mmu-miR-136-5p, mmu-miR-299-5p, mmu-miR-335-5p, mmu-miR-337, and mmu-miR-376c) were part of the two top clusters identified in Lamon et al. (2017). These clusters presented the largest changes over time and the highest biological significance, with "Cellular Development" and "Cellular Growth and Proliferation" being the highest ranked molecular and cellular functions associated with these clusters. The expression levels of these five miRNAs across the lifespan are depicted in Figure 5A. All five miRNAs not only presented 
a significant decrease with age (main effect, all $p<0.001$ ) but also continued to decrease in old muscle after adjustment for multiple comparisons (all $p<0.05$ ). Finally, the expression levels of lncMyoD1, Neat_v1, Neat_v2, Malat1, and lincmd1 displayed significant differences with time, although without an agingspecific pattern (Supplementary Figure 1).

\section{Target Analysis}

Target analysis identified three muscle-enriched genes that were potential targets of more than one miRNA of interest: Myf6 (hsamiR-299-5p, mmu-miR-434-3p, and mmu-miR-539-5p); Pax7 (mmu-miR-136-5p and mmu-miR-539-5p); and Mef2a (mmumiR-299-5p and mmu-miR-539-5p). The expression levels of these genes is shown in Supplementary Figure 2. Pax7 and Mef $2 a$ mRNA levels significantly decreased with aging, while expression Myf6 did not change, ruling out the hypothesis that the miRNAs identified in the screening may constitute primary negative regulators of their transcription.

\section{Gene and Protein Expression Levels of the Members of the DIk1-Dio3 Locus in Aging Quadriceps Muscle}

Expression levels of the lncRNAs Meg3, Mirg and Rian presented significant changes over the lifespan (main effect, $p<0.001$ ) (Figure 5B). Meg3 and Rian displayed further significant decreases between 12-week and 28-month of age after adjustment for multiple comparisons ( $p<0.05$ and $p<0.01$, respectively). The mRNA levels of Rtll and Dlk1 decreased by over 1000fold between 2-days and 28-months of age (all $p<0.001$ ),
A
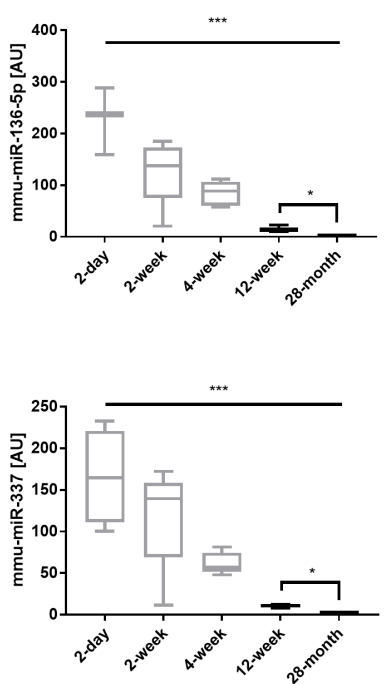

B

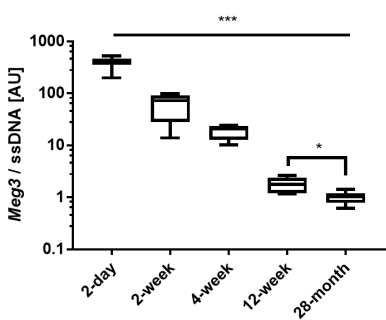

C

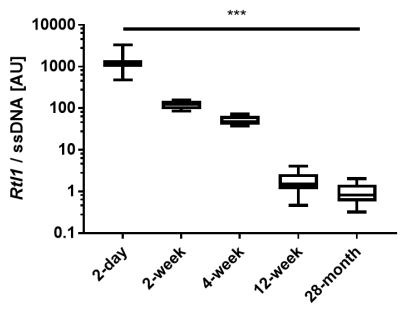

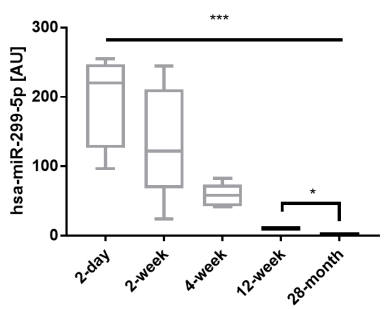
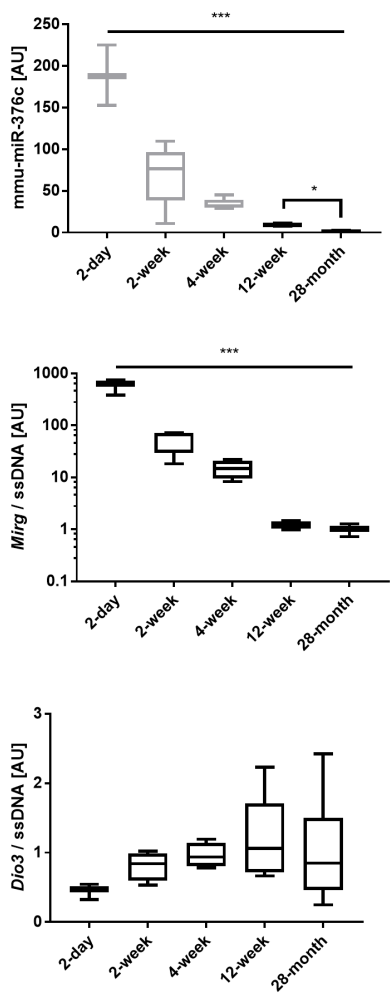
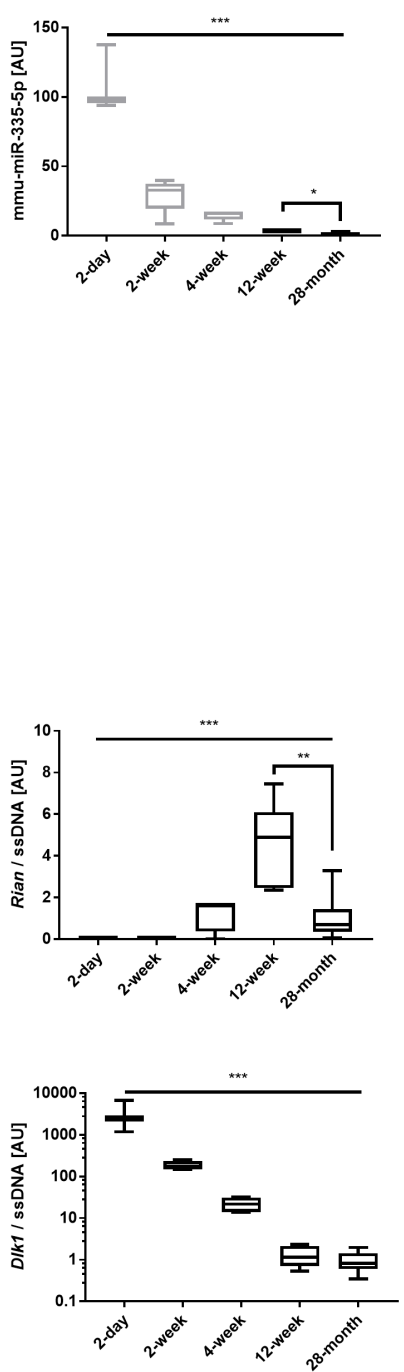

FIGURE 5 | Expression levels of miRNAs, IncRNAs, and mRNAs from the Callipyge locus over the lifespan (5 ages). (A) Individual miRNA expression levels of mmu-miR-136-5p, mmu-miR-299-5p, mmu-miR-335-5p, mmu-miR-337, and mmu-miR-376c over the lifespan. The data are reported as Mean \pm SEM. ***, main effect of time, $p<0.001$. The grayed data have been previously published in Lamon et al. (2017). (B) Individual expression levels of Meg3, Mirg, Rian, Rt/1, Dio3, and DIk1 over the lifespan. The data are reported as Mean \pm SEM. ${ }^{* * *}$, main effect of time, $p<0.001$. Post hoc tests: ${ }^{*} p<0.05$ and ${ }^{* *} p<0.01$. 
with no further reduction after the age of 12 weeks. Dio3 expression did not vary at any time point (Figure $5 \mathrm{C}$ ). In line with this result, DIO3 protein expression did not change with aging (Supplementary File S2 and Supplementary Figure 3A). The expression levels of the three other maternally expressed lncRNAs stemming from the mouse Dlk1-Dio3 region (namely anti-Rtl1, AK053394 and AK050713) was investigated. AntiRtl1 was not detected in adult mouse muscle, whilst AK053394 was expressed at levels that were hardly detectable by qPCR. In contrast, $A K 050713$ expression decreased by about 10 -fold between age 2 -week and 28-month $(p<0.001)$, with no further significant decreases between 12-week and 28-month of age (Supplementary Figure 3B).

\section{DISCUSSION}

MicroRNAs play a role in the regulation of skeletal muscle mass, metabolism and function over the lifespan and undergo high levels of regulation in skeletal muscle development and adaptation to health and disease conditions (Russell et al., 2013; Zacharewicz et al., 2014; Lamon et al., 2017; Butchart et al., 2018). More recently, the importance of lncRNAs in skeletal muscle growth and differentiation has started to emerge (Sunwoo et al., 2009; Cesana et al., 2011; Watts et al., 2013; Gong et al., 2015; Butchart et al., 2016). In two recently published studies, we investigated the expression patterns of 768 miRNAs and a series of selected lncRNAs in mouse quadriceps muscle between 2 days and 12 weeks of age (Butchart et al., 2016; Lamon et al., 2017). Following up on these results, the present study compared the expression levels of the same miRNAs and lncRNAs in mouse quadriceps muscle during early and very late (28 months) life stages. Out of the 12 miRNAs that were down-regulated with age, 10 clustered at the imprinted Dlk1-Dio3 domain-containing locus, also known as 'Callipyge', which controls important tissue homeostasis and pathogenesis processes (Benetatos et al., 2013). Coding (Dlk1 and Rtl1) and non-coding (Meg3, Mirg, and Rian) members of the Dlk1-Dio3 locus displayed 350-fold to 3500-fold decreases over the lifespan; however, only lncRNAs Meg3 and Rian kept significantly decreasing in adulthood between the age of 12 weeks and 28 months.

Several studies have reported large-scale miRNA profiling in aging mouse skeletal muscle. In some studies, the data were not adjusted for multiple testing (Hamrick et al., 2010; Kim et al., 2014), therefore presenting a high false discovery rate (Ranganathan et al., 2016) and suggesting that the results must be interpreted with caution. One study used nextgeneration sequencing (NGS) and identified 23 miRNAs that were differentially expressed in 6-month and 24-month TA muscle following statistical adjustment and qPCR validation (Jung et al., 2017). Another recent study conducted a fluorescence based miRNA array in 3- and 26-month old gastrocnemius muscle and used qPCR to confirm the differential expression of 14 miRNAs with aging (Pardo et al., 2017). In line with previous studies, our preliminary analysis demonstrated that individual miRNA expression can be positively or negatively regulated with aging. Following statistical adjustment, significant changes reflected a trend toward a collective down-regulation of miRNA expression with aging. One miRNA target, mmu-miR-434-3p, was consistently downregulated in other miRNA profiling studies in aging muscle (Hamrick et al., 2010; Kim et al., 2014; Jung et al., 2017; Pardo et al., 2017). Mmu-miR-434-3p directly targets the anti-apoptotic eukaryotic translation initiation factor 5A1 (Eif5a1) 3'UTR, suppresses its expression in mouse primary myotubes and protects the myotubes from apoptosis (Pardo et al., 2017). This suggests that, in vitro, mmu-miR-434-3p downregulation may contribute to age-related muscle atrophy by alleviating repression of Eif5a1 and allowing apoptotic processes to occur (Pardo et al., 2017). However, when observed in muscle tissue, apoptosis seems to mainly occur in the nuclei of interstitial cells, and whether these finding apply to whole muscle fibers in vivo has not been confirmed.

Ten of the 12 miRNAs that were significantly downregulated with aging clustered at the imprinted Dlk1-Dio3 genomic region (Schmidt et al., 2000), suggesting that this miRNA cluster is expressed in a polycistronic manner. This is in line with the findings from a previous study that observed a number of miRNAs dysegulated in aging gastrocnemius muscle at the same chromosomic location (Kim et al., 2014). The Dlk1-Dio3 genomic region, or 'Callipyge', is located on human chromosome 14 and mouse chromosome 12. The Callipyge locus was originally identified on ovine chromosome 18 (Cockett et al., 1994), where a mutation in sheep resulted in pronounced hypertrophy of the hindquarters muscles. The name is derived from the Greek calli- (beautiful) and pyge (buttocks). This chromosomal region contains three paternally expressed imprinted genes: delta like non-canonical Notch ligand 1 ( $D l k 1)$, retrotransposon Gaglike 1 (Rtll) and deiodinase, iodothyronine type III (Dio3), which are protein-coding; and four maternally expressed imprinted genes: maternally expressed 3 ( $M e g 3$ or Gtl2), RNA imprinted and accumulated in nucleus (Rian or Meg8), AK053394 and AK050713, which encode lncRNAs (Seitz et al., 2004; Glazov et al., 2008; Hagan et al., 2009). This locus also encodes Mirg (or Meg9), a large non-coding transcript that acts as a precursor for some of the 54 miRNAs (53 in the forward strand, 1 in the reverse strand) present in this region. This makes the Callipyge locus one of the largest miRNA clusters of the genome (Benetatos et al., 2013). In addition, an anti-Rtl1 transcript stems from the non-coding strand and is processed into miRNA precursors that display perfect complementarity with Rtl (Seitz et al., 2003; Davis et al., 2005). This cluster of miRNAs is dysregulated in pathological conditions including a wide range of cancers (Benetatos et al., 2013). A single-base mutation in the intergenic region located between $D l k 1$ and $M e g 3$ is responsible for the Callipyge trait (Freking et al., 2002), which, in sheep, translates to a $30-40 \%$ increase in muscle mass at the phenotypic level (Jackson et al., 1997). The Callipyge trait is only transmitted when the mutated allele comes from the father. This unusual, nonMendelian mode of inheritance is termed 'polar over dominance' (Cockett et al., 1996; Georges et al., 2003). The homozygous Callipyge genotype does not present a strong muscle phenotype (Cockett et al., 1996; Georges et al., 2003), and it has been suggested that a negative acting maternally expressed effector, potentially Mirg or anti-Rtl1, suppresses the paternal expression 
of Dlk1 or Rtl1 (Georges et al., 2003). Mice lacking either of the paternally imprinted genes Dlk1 (Moon et al., 2002), Rtl1 (Sekita et al., 2008), or Dio3 (Hernandez et al., 2006) display high rates of fetal deaths and/or severe growth defects. Interestingly, offspring born to mice lacking Glt2 only survive if they carry the double-deletion, where normal expression of the ncRNAs of the locus is restored (Takahashi et al., 2009). Rather than acting as general, non-locus regulators of gene and protein expression, these ncRNAs may therefore play an intricate role in the maintenance of gene regulation at the Dlk1-Dio3 locus itself.

Mouse muscle mass consistently decreases with aging (Barns et al., 2014; Soffe et al., 2016; White et al., 2016a,b), until the age of 24 months at least (Soffe et al., 2016). In our study, we observed large decreases in Meg3, AK050713, Mirg, Rtl1, and $D l k 1$ expression over the lifespan. However, past the age of 12 weeks, there were only decreases in the expression levels of Meg3 and Rian, suggesting the existence of upstream factors that individually regulate the transcription of the members of Dlk1-Dio3 locus. The Callipyge phenotype is evident when the mutation is paternally inherited by a heterozygous animal and is associated with increases in transcript abundance of Dlk1, Meg3, Rtll, and Rian in hypertrophied muscle (Bidwell et al., 2004). Dlk1 and Rtll expression is highest in the muscle of paternal heterozygous animals (Charlier et al., 2001; Bidwell et al., 2004), while Meg3 and Rian transcripts are almost exclusively found in the maternal heterozygous and homozygous animals (Charlier et al., 2001; Bidwell et al., 2004). Protein-coding Dlk1 and Rtl1 are proposed effectors for muscle hypertrophy and are potentially regulated by a negative acting maternally expressed effector such as Mirg or anti-Rtl1 (Georges et al., 2003). In line with this hypothesis, mice lacking the expression of the whole Dlk1-Dio3 miRNA cluster display hypertrophy of the fast-twitch muscle and elevated expression of the Dlk1 protein (Gao et al., 2015). Indeed, Dlk1 exclusively localizes in type II myofibers, which are significantly larger in sheep overexpressing Dlk1 than in their corresponding WT controls (White et al., 2008). Mice lacking the negative regulator of muscle growth myostatin also display increased expression of $D l k 1, M e g 3$, Rian, and Mirg, as well as a series of primary and mature forms of the miRNAs from the Callipyge locus (Hitachi and Tsuchida, 2017). The Dlk1-Dio3 locus might therefore act a positive regulator of muscle growth that may be directly or indirectly regulated by myostatin. In contrast, Dlk1 knockdown downregulated myosin heavy chain IIB gene expression, resulting in smaller muscles and reduced number of myofibers (Waddell et al., 2010). We therefore hypothesized that a downregulation of the Dlk1-Dio3 locus may contribute to age-related muscle atrophy, providing a potential new mechanism for sarcopenia.

Surprisingly and despite a striking downregulation of the whole miRNA cluster, we only observed decreases in the maternally expressed imprinted genes $M e g 3$ and Rian at the age of 28 months when compared with 12 weeks, and no changes in the paternally expressed imprinted protein-coding genes. Specifically, there was no change in Rtll or Dlkl gene expression with aging. MiR-127 and miR-136 are complementary to Rtl1 mRNA (Davis et al., 2005) and suppressing their expression induces an increase in Rtll expression (Seitz et al., 2003); an effect that we did not observe in our study despite close to 10fold decreases in the expression of these two miRNAs. Similarly, knocking out the imprinted Dlk1-Dio3 miRNA cluster results in elevated levels of the Dlk1 protein and muscle hypertrophy, leading to the hypothesis that maternal expression of the miRNA cluster may regulate paternal expression of $D l k 1$ (Gao et al., 2015). Again, and despite a strong collective dysregulation of at least 12 miRNAs of the Dlk1-Dio3 cluster, we did not observe an effect on Dlk1 gene expression levels. One possible explanation is that imprinting may not be rigorously maintained with age. For example, aging causes muscle transcriptome heterogeneity (Barns et al., 2014), which may result in partial loss of imprinting in some cells. The contribution of the Dlk1Dio3 locus to age-related muscle decline might therefore not be driven by the paternally imprinted Rtl1 or Dlk1. Future analyses investigating the methylation status at the imprinting control element and other long-range regulatory elements within the locus may confirm how age affects the methylation status of these sites.

Mmu-miR-335-5p was the only downregulated miRNA that was not part of the Dlk1-Dio3 genomic region. MiR335 harbors within an intron of the Mest gene, which is paternally imprinted. While miR-335 is highly expressed during muscle development and regeneration, miR-335, unlike Mest, is not necessary to normal muscle growth (Hiramuki et al., 2015). Supporting an age-induced decrease of miR-335-5p expression, we previously showed that hsa-miR-335-5p was downregulated in aging human muscle (Zacharewicz et al., 2014). MiR-335-5p was up-regulated in myotonic dystrophy type 1 (DM1) muscle samples (Perbellini et al., 2011), in Duchenne Muscular Dystrophy (DMD) (Greco et al., 2009) and in several other neuromuscular disorders (Eisenberg et al., 2007), suggesting a potential role for miR-335-5p in muscle adaptation in extreme physiological conditions involving continuous degeneration/regeneration cycles. This is supported by several lines of evidence showing that miR-335-5p directly targets genes of the transforming growth factor- $\beta$ (TGF- $\beta$ ) non-canonical pathways, such as mitogen-activated protein kinase 1 (MAPK1), resulting in reduced phosphorylation and inactivation of downstream pathway members (Lynch et al., 2012).

Finally, and despite significant changes during the early post-natal period confirming previous findings from our group (Butchart et al., 2016), changes in the expression levels of lncMyoD1, Neat_v1, Neat_v2, Malat1, and Lincmd1 did not persist past the age of 12 weeks. These lncRNAs may therefore play an important regulatory role during hyperplasia and early hypertrophic growth and maturation, rather than in age-related muscle decline.

In conclusion, our study examined a combination of short and lncRNAs in old mouse skeletal muscle. We confirm that the collective decrease in muscle miRNA expression observed at early post-natal stages persists until old age, while muscle-specific lncRNAs display little regulation with aging. An unexpected finding was that the vast majority of the miRNA and lncRNA targets displaying significant decreases with aging were related to the Callipyge locus, which is associated with muscle development 
and hypertrophy. The dysregulation of this locus in aging muscle may therefore represent a potential new mechanism for agerelated muscle decline.

\section{AVAILABILITY OF DATA AND MATERIAL}

All data and material that are not presented in the main paper or supplementary files are available from the corresponding author on request.

\section{AUTHOR CONTRIBUTIONS}

SL, JM, and MG designed the study. SL and LB provided the samples. JM, KS, FG-R, and PDG collected the data. SL and SV analyzed the data and completed the statistical analysis. SL and MG funded the study. All authors contributed to manuscript writing and editing.

\section{FUNDING}

SL was supported by a Discovery Early Career Research Award (DECRA) from the Australian Research Council (ARC)

\section{REFERENCES}

Asp, P., Blum, R., Vethantham, V., Parisi, F., Micsinai, M., Cheng, J., et al. (2011). Genome-wide remodeling of the epigenetic landscape during myogenic differentiation. Proc. Natl. Acad. Sci. U.S.A. 108, E149-E158. doi: 10.1073/pnas. 1102223108

Barns, M., Gondro, C., Tellam, R. L., Radley-Crabb, H. G., Grounds, M. D., and Shavlakadze, T. (2014). Molecular analyses provide insight into mechanisms underlying sarcopenia and myofibre denervation in old skeletal muscles of mice. Int. J. Biochem. Cell Biol. 53, 174-185. doi: 10.1016/j.biocel.2014.04.025

Bartel, D. P. (2004). MicroRNAs: genomics, biogenesis, mechanism, and function. Cell 116, 281-297. doi: 10.1016/S0092-8674(04)00045-5

Benetatos, L., Hatzimichael, E., Londin, E., Vartholomatos, G., Loher, P., Rigoutsos, I., et al. (2013). The microRNAs within the DLK1-DIO3 genomic region: involvement in disease pathogenesis. Cell Mol. Life Sci. 70, 795-814. doi: 10.1007/s00018-012-1080-8

Bidwell, C. A., Kramer, L. N., Perkins, A. C., Hadfield, T. S., Moody, D. E., and Cockett, N. E. (2004). Expression of PEG11 and PEG11AS transcripts in normal and callipyge sheep. BMC Biol. 2:17. doi: 10.1186/1741-7007-2-17

Birben, E., Sahiner, U. M., Sackesen, C., Erzurum, S., and Kalayci, O. (2012). Oxidative stress and antioxidant defense. World Allergy Organ. J. 5, 9-19.

Butchart, L. C., Fox, A., Shavlakadze, T., and Grounds, M. D. (2016). The long and short of non-coding RNAs during post-natal growth and differentiation of skeletal muscles: focus on lncRNA and miRNAs. Differentiation 92, 237-248. doi: 10.1016/j.diff.2016.05.003

Butchart, L. C., Terrill, J. R., Rossetti, G., White, R., Filipovska, A., and Grounds, M. D. (2018). Expression patterns of regulatory RNAs, including lncRNAs and tRNAs, during postnatal growth of normal and dystrophic ( $\mathrm{mdx}$ ) mouse muscles, and their response to taurine treatment. Int. J. Biochem. Cell Biol. 99, 52-63. doi: 10.1016/j.biocel.2018.03.016

Carey, K. A., Farnfield, M. M., Tarquinio, S. D., and Cameron-Smith, D. (2007). Impaired expression of Notch signaling genes in aged human skeletal muscle. J. Gerontol. A Biol. Sci. Med. Sci. 62, 9-17. doi: 10.1093/gerona/ 62.1 .9

Cesana, M., Cacchiarelli, D., Legnini, I., Santini, T., Sthandier, O., Chinappi, M., et al. (2011). A long noncoding RNA controls muscle differentiation
(DE150100538). JM was supported by a Deakin University Post-graduate Scholarship (DUPR). LB was supported by international Ph.D. scholarships from the University of Western Australia (UPAIS, SIRF, and UWA Top-up Scholarship). The research at UWA was supported by the Duchenne Parent Project the Netherlands. None of the funding bodies had any role in the design of the study, the collection, analysis, and interpretation of data and the writing of the manuscript.

\section{ACKNOWLEDGMENTS}

We thank Robyn Murphy for her generous contribution to the maintenance of the 28-month old mouse cohort. We also thank Ross Tellam for his expert advice on the Callipyge locus.

\section{SUPPLEMENTARY MATERIAL}

The Supplementary Material for this article can be found online at: https://www.frontiersin.org/articles/10.3389/fgene. 2018.00548/full\#supplementary-material

by functioning as a competing endogenous RNA. Cell 147, 358-369. doi: 10.1016/j.cell.2011.09.028

Chai, R. J., Vukovic, J., Dunlop, S., Grounds, M. D., and Shavlakadze, T. (2011). Striking denervation of neuromuscular junctions without lumbar motoneuron loss in geriatric mouse muscle. PLoS One 6:e28090. doi: 10.1371/journal.pone. 0028090

Charlier, C., Segers, K., Karim, L., Shay, T., Gyapay, G., Cockett, N., et al. (2001). The callipyge mutation enhances the expression of coregulated imprinted genes in cis without affecting their imprinting status. Nat. Genet. 27, 367-369. doi: $10.1038 / 86856$

Cockett, N. E., Jackson, S. P., Shay, T. L., Farnir, F., Berghmans, S., Snowder, G. D., et al. (1996). Polar overdominance at the ovine callipyge locus. Science 273, 236-238. doi: 10.1126/science.273.5272.236

Cockett, N. E., Jackson, S. P., Shay, T. L., Nielsen, D., Moore, S. S., Steele, M. R., et al. (1994). Chromosomal localization of the callipyge gene in sheep (Ovis aries) using bovine DNA markers. Proc. Natl. Acad. Sci. U.S.A. 91, 3019-3023. doi: 10.1073/pnas.91.8.3019

Combaret, L., Dardevet, D., Rieu, I., Pouch, M. N., Bechet, D., Taillandier, D., et al. (2005). A leucine-supplemented diet restores the defective postprandial inhibition of proteasome-dependent proteolysis in aged rat skeletal muscle. J. Physiol. 569(Pt 2), 489-499. doi: 10.1113/jphysiol.2005.09 8004

Davis, E., Caiment, F., Tordoir, X., Cavaille, J., Ferguson-Smith, A., Cockett, N., et al. (2005). RNAi-mediated allelic trans-interaction at the imprinted Rtl1/Peg11 locus. Curr. Biol. 15, 743-749. doi: 10.1016/j.cub.2005.02.060

Eisenberg, I., Eran, A., Nishino, I., Moggio, M., Lamperti, C., Amato, A. A., et al. (2007). Distinctive patterns of microRNA expression in primary muscular disorders. Proc. Natl. Acad. Sci. U.S.A. 104, 17016-17021. doi: 10.1073/pnas. 0708115104

Engreitz, J. M., Ollikainen, N., and Guttman, M. (2016). Long non-coding RNAs: spatial amplifiers that control nuclear structure and gene expression. Nat. Rev. Mol. Cell Biol. 17:756. doi: 10.1038/nrm.2016.126

Freking, B. A., Murphy, S. K., Wylie, A. A., Rhodes, S. J., Keele, J. W., Leymaster, K. A., et al. (2002). Identification of the single base change causing the callipyge muscle hypertrophy phenotype, the only known example of polar overdominance in mammals. Genome Res. 12, 1496-1506. doi: 10.1101/gr. 571002 
Fujimaki, S., Wakabayashi, T., Takemasa, T., Asashima, M., and Kuwabara, T. (2015). The regulation of stem cell aging by Wnt signaling. Histol. Histopathol. 30, 1411-1430. doi: 10.14670/hh-11-657

Gao, Y. Q., Chen, X., Wang, P., Lu, L., Zhao, W., Chen, C., et al. (2015). Regulation of DLK1 by the maternally expressed miR-379/miR-544 cluster may underlie callipyge polar overdominance inheritance. Proc. Natl. Acad. Sci. U.S.A. 112, 13627-13632. doi: 10.1073/pnas. 1511448112

Georges, M., Charlier, C., and Cockett, N. (2003). The callipyge locus: evidence for the trans interaction of reciprocally imprinted genes. Trends Genet. 19, 248-252. doi: 10.1016/s0168-9525(03)00082-9

Glazov, E. A., McWilliam, S., Barris, W. C., and Dalrymple, B. P. (2008). Origin, evolution, and biological role of miRNA cluster in DLK-DIO3 genomic region in placental mammals. Mol. Biol. Evol. 25, 939-948. doi: 10.1093/molbev/ msn045

Gong, C., Li, Z., Ramanujan, K., Clay, I., Zhang, Y., Lemire-Brachat, S., et al. (2015). A long non-coding RNA, LncMyoD, regulates skeletal muscle differentiation by blocking IMP2-mediated mRNA translation. Dev. Cell 34, 181-191. doi: 10.1016/. devcel.2015.05.009

Greco, S., Simone, M. D., Colussi, C., Zaccagnini, G., Fasanaro, P., Pescatori, M., et al. (2009). Common micro-RNA signature in skeletal muscle damage and regeneration induced by Duchenne muscular dystrophy and acute ischemia. FASEB J. 23, 3335-3346. doi: 10.1096/fj.08-128579

Grounds, M. D. (2014). Therapies for sarcopenia and regeneration of old skeletal muscles: more a case of old tissue architecture than old stem cells. Bioarchitecture 4, 81-87. doi: 10.4161/bioa.29668

Hagan, J. P., O'Neill, B. L., Stewart, C. L., Kozlov, S. V., and Croce, C. M. (2009). At least ten genes define the imprinted Dlk1-Dio3 cluster on mouse chromosome 12qF1. PLoS One 4:e4352. doi: 10.1371/journal.pone.0004352

Hamrick, M. W., Herberg, S., Arounleut, P., He, H. Z., Shiver, A., Qi, R. Q., et al. (2010). The adipokine leptin increases skeletal muscle mass and significantly alters skeletal muscle miRNA expression profile in aged mice. Biochem. Biophys. Res. Commun. 400, 379-383. doi: 10.1016/j.bbrc.2010.08.079

Hernandez, A., Martinez, M. E., Fiering, S., Galton, V. A., and St Germain, D. (2006). Type 3 deiodinase is critical for the maturation and function of the thyroid axis. J. Clin. Invest. 116, 476-484. doi: 10.1172/jci 26240

Hiramuki, Y., Sato, T., Furuta, Y., Surani, M. A., and Sehara-Fujisawa, A. (2015). Mest but not MiR-335 affects skeletal muscle growth and regeneration. PLoS One 10:e0130436. doi: 10.1371/journal.pone.0130436

Hitachi, K., and Tsuchida, K. (2017). Myostatin-deficiency in mice increases global gene expression at the Dlk1-Dio3 locus in the skeletal muscle. Oncotarget 8, 5943-5953. doi: 10.18632/oncotarget.13966

Howlett, K. F., and McGee, S. L. (2016). Epigenetic regulation of skeletal muscle metabolism. Clin. Sci. 130, 1051-1063. doi: 10.1042/cs2016 0115

Huang, Z. P., Espinoza-Lewis, R., and Wang, D. Z. (2012). Determination of miRNA targets in skeletal muscle cells. Methods Mol. Biol. 798, 475-490. doi: 10.1007/978-1-61779-343-1_28

Jackaman, C., Radley-Crabb, H. G., Soffe, Z., Shavlakadze, T., Grounds, M. D., and Nelson, D. J. (2013). Targeting macrophages rescues age-related immune deficiencies in C57BL/6J geriatric mice. Aging Cell 12, 345-357. doi: 10.1111/ acel.12062

Jackson, S. P., Miller, M. F., and Green, R. D. (1997). Phenotypic characterization of rambouillet sheep expression the callipyge gene: III. Muscle weights and muscle weight distribution. J. Anim. Sci. 75, 133-138. doi: 10.2527/1997.751133x

Johnsson, P., Lipovich, L., Grander, D., and Morris, K. V. (2014). Evolutionary conservation of long non-coding RNAs; sequence, structure, function. Biochim. Biophys. Acta 1840, 1063-1071. doi: 10.1016/j.bbagen.2013.10.035

Jung, H. J., Lee, K. P., Milholland, B., Shin, Y. J., Kang, J. S., Kwon, K. S., et al. (2017). Comprehensive miRNA profiling of skeletal muscle and serum in induced and normal mouse muscle atrophy during aging. J. Gerontol. A Biol. Sci. Med. Sci. 72, 1483-1491. doi: 10.1093/gerona/glx025

Kim, J. Y., Park, Y. K., Lee, K. P., Lee, S. M., Kang, T. W., Kim, H. J., et al. (2014). Genome-wide profiling of the microRNA-mRNA regulatory network in skeletal muscle with aging. Aging 6, 524-544. doi: 10.18632/aging.100677

Kirby, T. J., and McCarthy, J. J. (2013). MicroRNAs in skeletal muscle biology and exercise adaptation. Free Radic. Biol. Med. 64, 95-105. doi: 10.1016/j. freeradbiomed.2013.07.004
Kovanda, A., Rezen, T., and Rogelj, B. (2014). MicroRNA in skeletal muscle development, growth, atrophy, and disease. Wiley Interdiscip. Rev. RNA 5, 509-525. doi: 10.1002/wrna.1227

Lamon, S., Zacharewicz, E., Butchart, L. C., Orellana, L., Mikovic, J., Grounds, M. D., et al. (2017). MicroRNA expression patterns in post-natal mouse skeletal muscle development. BMC Genomics 18:52. doi: 10.1186/s12864-016-3399-2

Lexell, J., Taylor, C. C., and Sjöström, M. (1988). What is the cause of the ageing atrophy? Total number, size and proportion of different fiber types studied in whole vastus lateralis muscle from 15- to 83-year-old men. J. Neurol. Sci. 84, 275-294. doi: 10.1016/0022-510X(88)90132-3

Lynch, J., Fay, J., Meehan, M., Bryan, K., Watters, K. M., Murphy, D. M., et al. (2012). MiRNA-335 suppresses neuroblastoma cell invasiveness by direct targeting of multiple genes from the non-canonical TGF-beta signalling pathway. Carcinogenesis 33, 976-985. doi: 10.1093/carcin/bgs114

McCarthy, J. J. (2008). MicroRNA-206: the skeletal muscle-specific myomiR. Biochim. Biophys. Acta 1779, 682-691. doi: 10.1016/j.bbagrm.2008.03.001

Moon, Y. S., Smas, C. M., Lee, K., Villena, J. A., Kim, K. H., Yun, E. J., et al. (2002). Mice lacking paternally expressed Pref-1/Dlk1 display growth retardation and accelerated adiposity. Mol. Cell. Biol. 22, 5585-5592. doi: 10.1128/MCB.22.15. 5585-5592.2002

Neguembor, M. V., Jothi, M., and Gabellini, D. (2014). Long noncoding RNAs, emerging players in muscle differentiation and disease. Skelet. Muscle 4:8. doi: 10.1186/2044-5040-4-8

Neppl, R. L., Wu, C.-L., and Walsh, K. (2017). IncRNA Chronos is an aginginduced inhibitor of muscle hypertrophy. J. Cell Biol. 216, 3497-3507. doi: $10.1083 /$ jcb. 201612100

Pardo, P. S., Hajira, A., Boriek, A. M., and Mohamed, J. S. (2017). MicroRNA-434$3 \mathrm{p}$ regulates age-related apoptosis through eIF5A1 in the skeletal muscle. Aging 9, 1012-1029. doi: 10.18632/aging.101207

Perbellini, R., Greco, S., Sarra-Ferraris, G., Cardani, R., Capogrossi, M. C., Meola, G., et al. (2011). Dysregulation and cellular mislocalization of specific miRNAs in myotonic dystrophy type 1. Neuromuscul. Disord. 21, 81-88. doi: 10.1016/j.nmd.2010.11.012

Ranganathan, P., Pramesh, C. S., and Buyse, M. (2016). Common pitfalls in statistical analysis: the perils of multiple testing. Perspect. Clin. Res. 7, 106-107. doi: 10.4103/2229-3485.179436

Rieu, I., Balage, M., Sornet, C., Giraudet, C., Pujos, E., Grizard, J., et al. (2006). Leucine supplementation improves muscle protein synthesis in elderly men independently of hyperaminoacidaemia. J. Physiol. 575(Pt 1), 305-315. doi: 10.1113/jphysiol.2006.110742

Rooyackers, O. E., Adey, D. B., Ades, P. A., and Nair, K. S. (1996). Effect of age on in vivo rates of mitochondrial protein synthesis in human skeletal muscle. Proc. Natl. Acad. Sci. U.S.A. 93, 15364-15369. doi: 10.1073/pnas.93.26.15364

Russell, A. P., Ghobrial, L., Ngo, S., Yerbury, J., Zacharewicz, E., Chung, R., et al. (2017). Dysregulation of microRNA biogenesis machinery and microRNA/RNA ratio in skeletal muscle of amyotrophic lateral sclerosis mice. Muscle Nerve 57, 838-847. doi: 10.1002/mus.26039

Russell, A. P., Wada, S., Vergani, L., Hock, M. B., Lamon, S., Leger, B., et al. (2013). Disruption of skeletal muscle mitochondrial network genes and miRNAs in amyotrophic lateral sclerosis. Neurobiol. Dis. 49, 107-117. doi: 10.1016/j.nbd. 2012.08.015

Sayer, A. A., Robinson, S. M., Patel, H. P., Shavlakadze, T., Cooper, C., and Grounds, M. D. (2013). New horizons in the pathogenesis, diagnosis and management of sarcopenia. Age Ageing 42, 145-150. doi: 10.1093/ageing/afs191

Schmidt, J. V., Matteson, P. G., Jones, B. K., Guan, X. J., and Tilghman, S. M. (2000). The Dlk1 and Gt12 genes are linked and reciprocally imprinted. Genes Dev. 14, 1997-2002.

Seitz, H., Royo, H., Bortolin, M. L., Lin, S. P., Ferguson-Smith, A. C., and Cavaille, J. (2004). A large imprinted microRNA gene cluster at the mouse Dlk1-Gtl2 domain. Genome Res. 14, 1741-1748. doi: 10.1101/gr.2743304

Seitz, H., Youngson, N., Lin, S. P., Dalbert, S., Paulsen, M., Bachellerie, J. P., et al. (2003). Imprinted microRNA genes transcribed antisense to a reciprocally imprinted retrotransposon-like gene. Nat. Genet. 34, 261-262. doi: 10.1038/ ng1171

Sekita, Y., Wagatsuma, H., Nakamura, K., Ono, R., Kagami, M., Wakisaka, N., et al. (2008). Role of retrotransposon-derived imprinted gene, Rtll, in the fetomaternal interface of mouse placenta. Nat. Genet. 40, 243-248. doi: 10.1038/ng. 2007.51 
Short, K. R., Bigelow, M. L., Kahl, J., Singh, R., Coenen-Schimke, J., Raghavakaimal, S., et al. (2005). Decline in skeletal muscle mitochondrial function with aging in humans. Proc. Natl. Acad. Sci. U.S.A. 102, 5618-5623. doi: 10.1073/pnas.0501559102

Soffe, Z., Radley-Crabb, H. G., McMahon, C., Grounds, M. D., and Shavlakadze, T. (2016). Effects of loaded voluntary wheel exercise on performance and muscle hypertrophy in young and old male C57Bl/6J mice. Scand. J. Med. Sci. Sports 26, 172-188. doi: 10.1111/sms.12416

Stearns-Reider, K. M., D’Amore, A., Beezhold, K., Rothrauff, B., Cavalli, L., Wagner, W. R., et al. (2017). Aging of the skeletal muscle extracellular matrix drives a stem cell fibrogenic conversion. Aging Cell 16, 518-528. doi: 10.1111/acel. 12578

Sunwoo, H., Dinger, M. E., Wilusz, J. E., Amaral, P. P., Mattick, J. S., and Spector, D. L. (2009). MEN $\varepsilon / \beta$ nuclear-retained non-coding RNAs are up-regulated upon muscle differentiation and are essential components of paraspeckles. Genome Res. 19, 347-359. doi: 10.1101/gr.087775.108

Takahashi, N., Okamoto, A., Kobayashi, R., Shirai, M., Obata, Y., Ogawa, H., et al. (2009). Deletion of Gtl2, imprinted non-coding RNA, with its differentially methylated region induces lethal parent-origin-dependent defects in mice. Hum. Mol. Genet. 18, 1879-1888. doi: 10.1093/hmg/ddp108

Tohma, H., El-Shafey, A. F., Croft, K., Shavlakadze, T., Grounds, M. D., and Arthur, P. G. (2014). Protein thiol oxidation does not change in skeletal muscles of aging female mice. Biogerontology 15, 87-98. doi: 10.1007/s10522-0139483-y

von Maltzahn, J., Chang, N. C., Bentzinger, C. F., and Rudnicki, M. A. (2012). Wnt signaling in myogenesis. Trends Cell Biol. 22, 602-609. doi: 10.1016/j.tcb.2012. 07.008

Waddell, J. N., Zhang, P., Wen, Y., Gupta, S. K., Yevtodiyenko, A., Schmidt, J. V., et al. (2010). Dlk1 is necessary for proper skeletal muscle development and regeneration. PLoS One 5:e15055. doi: 10.1371/journal.pone.0015055

Wang, K. C., and Chang, H. Y. (2011). Molecular mechanisms of long noncoding RNAs. Mol. Cell. 43, 904-914. doi: 10.1016/j.molcel.2011.08.018

Watts, R., Johnsen, V. L., Shearer, J., and Hittel, D. S. (2013). Myostatin-induced inhibition of the long noncoding RNA Malat1 is associated with decreased myogenesis. Am. J. Physiol. Cell Physiol. 304, C995-C1001. doi: 10.1152/ajpcell. 00392.2012

White, J. D., Vuocolo, T., McDonagh, M., Grounds, M. D., Harper, G. S., Cockett, N. E., et al. (2008). Analysis of the callipyge phenotype through skeletal muscle development; association of Dlk1 with muscle precursor cells. Differentiation 76, 283-298. doi: 10.1111/j.1432-0436.2007.00208.x

White, Z., Terrill, J., White, R. B., McMahon, C., Sheard, P., Grounds, M. D., et al. (2016a). Voluntary resistance wheel exercise from mid-life prevents sarcopenia and increases markers of mitochondrial function and autophagy in muscles of old male and female C57BL/6J mice. Skelet. Muscle 6:45. doi: 10.1186/s13395016-0117-3

White, Z., White, R. B., McMahon, C., Grounds, M. D., and Shavlakadze, T. (2016b). High mTORC1 signaling is maintained, while protein degradation pathways are perturbed in old murine skeletal muscles in the fasted state. Int. J. Biochem. Cell Biol. 78, 10-21. doi: 10.1016/j.biocel.2016.06.012

Yin, H., Price, F., and Rudnicki, M. A. (2013). Satellite cells and the muscle stem cell niche. Physiol. Rev. 93, 23-67. doi: 10.1152/physrev.00043. 2011

Yun, M. H. (2015). Changes in Regenerative Capacity through Lifespan. Int. J. Mol. Sci. 16, 25392-25432. doi: 10.3390/ijms161025392

Zacharewicz, E., Della Gatta, P., Reynolds, J., Garnham, A., Crowley, T., Russell, A. P., et al. (2014). Identification of microRNAs linked to regulators of muscle protein synthesis and regeneration in young and old skeletal muscle. PLoS One 9:e114009. doi: 10.1371/journal.pone.0114009

Zhao, Y., and Srivastava, D. (2007). A developmental view of microRNA function. Trends Biochem. Sci. 32, 189-197. doi: 10.1016/j.tibs.2007.02.006

Zong-Kang, Z., Jie, L., Daogang, G., Chao, L., Zhenjian, Z., Jin, L., et al. (2018). A newly identified lncRNA MAR1 acts as a miR-487b sponge to promote skeletal muscle differentiation and regeneration. J. Cachexia Sarcopenia Muscle 9, 613-626. doi: 10.1002/jcsm.12281

Conflict of Interest Statement: The authors declare that the research was conducted in the absence of any commercial or financial relationships that could be construed as a potential conflict of interest.

Copyright (® 2018 Mikovic, Sadler, Butchart, Voisin, Gerlinger-Romero, Della Gatta, Grounds and Lamon. This is an open-access article distributed under the terms of the Creative Commons Attribution License (CC BY). The use, distribution or reproduction in other forums is permitted, provided the original author(s) and the copyright owner(s) are credited and that the original publication in this journal is cited, in accordance with accepted academic practice. No use, distribution or reproduction is permitted which does not comply with these terms. 